\title{
CIRCLE AND TORUS ACTIONS ON EQUAL SYMPLECTIC BLOW-UPS OF $\mathrm{CP}^{2}$
}

\author{
Yael Karshon and Liat Kessler
}

\begin{abstract}
A manifold obtained by $k$ simultaneous symplectic blow-ups of $\mathbb{C P}^{2}$ of equal sizes $\epsilon$ (where the size of $\mathbb{C P}^{1} \subset \mathbb{C P}^{2}$ is one) admits an effective two dimensional torus action if $k \leq 3$ and admits an effective circle action if $\epsilon<1 /(k-1)$. We show that these bounds are sharp if $\epsilon=1 / n$ where $n$ is a natural number. Our proof combines "soft" equivariant techniques with "hard" holomorphic techniques.
\end{abstract}

\section{Toric actions and circle actions in dimension four}

Hamiltonian torus actions. Let a torus $T \cong\left(S^{1}\right)^{k}$ act effectively on a compact connected symplectic manifold $(M, \omega)$ of dimension $2 n$ by symplectic transformations. The action is Hamiltonian if there exists a moment map, that is, a map

$$
\Phi: M \rightarrow \mathfrak{t}^{*} \cong \mathbb{R}^{k}
$$

such that

$$
d \Phi_{j}=-\iota\left(\xi_{j}\right) \omega
$$

for all $j=1, \ldots, k$, where $\xi_{1}, \ldots, \xi_{k}$ are the vector fields that generate the torus action. The triple $(M, \omega, \Phi)$ is then called a Hamiltonian T-manifold. If $H^{1}(M)=0$ then every symplectic torus action is Hamiltonian. By the convexity theorem [At, GS1], the image of the moment map,

$$
\Delta:=\Phi(M),
$$

is a convex polytope. By the equivariant Darboux-Weinstein theorem [W], every $T$-fixed point has a neighborhood $U$ which is equivariantly symplectomorphic to a neighborhood of the origin in $\mathbb{C}^{n}$ with $T$ acting linearly. The components $\Phi^{\xi}=\langle\Phi, \xi\rangle$, $\xi \in \mathfrak{t}$, of the moment map are perfect Morse-Bott functions; see [GS1].

The Delzant theorem. If $\operatorname{dim} T=\frac{1}{2} \operatorname{dim} M$, the triple $(M, \omega, \Phi)$ is a symplectic toric manifold, and the $T$-action is called toric. The polytope $\Delta$ determines $(M, \omega, \Phi)$ up to an equivariant symplectomorphism preserving $\Phi$. (This is the Delzant theorem [De]; also see [LT].) The inverse image under $\Phi$ of a vertex of $\Delta$ is a fixed point for the $T$-action, and the image of a $T$-fixed point is a vertex of $\Delta$.

A necessary condition for $\Delta$ to occur as the moment map image of a symplectic toric manifold is that it be a Delzant polytope, meaning that the edges emanating from each vertex are generated by vectors $v_{1}, \ldots, v_{n}$ that span the lattice $\mathbb{Z}^{n}$.

Received by the editors June 17, 2005. Revision received June 20, 2007.

2000 Mathematics Subject Classification. Primary 53D20, 53D35, 53D45. Secondary 57S15. 
Delzant's construction. Given a Delzant polytope $\Delta$, Delzant constructs a symplectic toric manifold $\left(M_{\Delta}, \omega_{\Delta}, \Phi_{\Delta}\right)$ whose moment map image is $\Delta$. This manifold is a symplectic quotient of $\mathbb{C}^{N}$, where $N$ is the number of facets of $\Delta$, with respect to a subgroup $K$ of $\left(S^{1}\right)^{N}$. The toric $T$-action is through an isomorphism of $T$ with the quotient $\left(S^{1}\right)^{N} / K$. The polytope $\Delta$ is then realized as the intersection of the positive orthant $\mathbb{R}_{+}^{N}$ with an affine plane. See $[\mathrm{De}],[\mathrm{Au}]$, or $[\mathrm{G}]$.

Toric actions on $\mathbf{C P}^{2}$. An important special case of the Delzant construction is the construction of $\mathbb{C P}^{2}$ as the quotient of the sphere $\left\{\left|z_{1}\right|^{2}+\left|z_{2}\right|^{2}+\left|z_{3}\right|^{2}=2\right\} \subset \mathbb{C}^{3}$ by the diagonal $S^{1}$-action, resulting in the Fubini-Study symplectic form $\omega_{\mathrm{FS}}$ on $\mathbb{C P}^{2}$. Our normalization is so that $\frac{1}{2 \pi} \int_{\mathbb{C P}^{1}} \omega_{\mathrm{FS}}=1$. Whenever we refer to $\mathbb{C P}^{2}$ as a symplectic manifold, we assume that the symplectic form is $\omega_{\mathrm{FS}}$.

The standard toric action on $\mathbb{C P}^{2}$ is $(a, b) \cdot\left[z_{0}: z_{1}: z_{2}\right]=\left[z_{0}: a z_{1}: b z_{2}\right]$. The moment map image is the triangle

$$
\left\{\left(x_{1}, x_{2}\right) \in \mathbb{R}^{2} \mid x_{1} \geq 0, x_{2} \geq 0, x_{1}+x_{2} \leq 1\right\} .
$$

By the Delzant theorem, every toric action on $\mathbb{C P}^{2}$ is obtained from a symplectomorphism of $\mathbb{C P}^{2}$ with a symplectic toric manifold $M_{\Delta}$ that is associated to a Delzant polygon $\Delta$. The second Betti number $\operatorname{dim} H_{2}\left(M_{\Delta}\right)$ is equal to the number of edges of $\Delta$ minus two; this follows from Morse theory for components of the moment map. So $\Delta$ must be a triangle. Up to rescaling, every Delzant triangle can be obtained from a standard one, (1.1), by a transformation $x \mapsto A x+b$ where $A \in \mathrm{GL}(2, \mathbb{Z})$ and $b \in \mathbb{R}^{2}$. It follows that

Lemma 1.1. Every toric T-action on a compact connected symplectic four-manifold $(M, \omega)$ with $\operatorname{dim} H_{2}(M)=1$ and volume $(M)=$ volume $\left(\mathbb{C P}^{2}\right)$ is equivariantly symplectomorphic to the standard action on $\mathbb{C P}^{2}$ through an isomorphism of the torus $T$ with $\left(S^{1}\right)^{2}$.

Hamiltonian $\mathbf{S}^{\mathbf{1}}$ actions on compact symplectic four-manifolds. Let the circle group $T=S^{1}$ act on a four dimensional compact connected symplectic manifold $(M, \omega)$ with moment map $\Phi: M \rightarrow \mathfrak{t}^{*} \cong \mathbb{R}$. A connected component of the fixed point set is either an isolated fixed point $p \in M$ or a closed symplectic surface $F \subset M$ on which $\Phi$ is constant. Every interior fixed point (that is, a fixed point at which $\Phi$ is neither maximal nor minimal) is isolated. Let $\mathbf{Z}_{k} \subset S^{1}$ be the cyclic subgroup of order $k$. A connected component of the fixed point set of $\mathbf{Z}_{k}$ that is not fixed by any larger subgroup is a symplectic 2-sphere $C \subset M$ on which $S^{1}$ acts by rotations of "speed k"; we call it a $\mathbf{Z}_{k}$-sphere. A $\mathbf{Z}_{k}$-sphere contains two fixed points, $p$ and $q$, which are isolated fixed points in $M$. We have $\frac{1}{k}|\Phi(p)-\Phi(q)|=\frac{1}{2 \pi} \int_{C} \omega$.

To $(M, \omega, \Phi)$ we associate the following labeled graph. To an isolated fixed point $p$ we associate a vertex $\langle p\rangle$, labeled by the real number $\Phi(p)$. To a $\mathbf{Z}_{k}$-sphere containing two fixed points $p$ and $q$ we associate an edge connecting the vertices $\langle p\rangle$ and $\langle q\rangle$ and labeled by the integer $k$. To a two dimensional component $F$ of the fixed point set we associate a "fat" vertex $\langle F\rangle$ labeled by two real numbers and one integer: the moment map label $\Phi(F)$, the area label $\frac{1}{2 \pi} \int_{F} \omega$, and the genus of the surface $F$. The graph determines $(M, \omega, \Phi)$ up to an equivariant symplectomorphism that preserves moment maps [Ka1]. 
Circle actions on $\mathbf{C P}^{2}$. Let $(M, \omega)$ be a compact symplectic four-manifold. If $\operatorname{dim} H^{2}(M ; \mathbb{R}) \leq 3$ and $\operatorname{dim} H^{1}(M ; \mathbb{R})=0$, then every Hamiltonian circle action on $(M, \omega)$ extends to a toric action. This is proved in [Ka2, Theorem 1] using techniques of [Ka1]. In particular,

Lemma 1.2. Every Hamiltonian $S^{1}$-action on a compact connected symplectic fourmanifold $(M, \omega)$ with $\operatorname{dim} H_{2}(M)=1$ and volume $(M)=$ volume $\left(\mathbb{C P}^{2}\right)$ is equivariantly symplectomorphic to an $S^{1}$-action on $\mathbb{C P}^{2}$ obtained by composing a homomorphism $S^{1} \rightarrow\left(S^{1}\right)^{2}$ with the standard toric action on $\mathbb{C P}^{2}$.

\section{Symplectic blow-ups}

Blow-up of $\mathbf{C}^{\mathbf{n}}$. Consider $\mathbb{C}^{n} \cong \mathbb{R}^{2 n}$ with its standard symplectic form $\sum d x_{i} \wedge d y_{i}$. The standard symplectic blow-up of $\mathbb{C}^{n}$ of size $r^{2} / 2$ is obtained by removing the open ball $B^{2 n}(r)$ of radius $r$ centered at the origin and collapsing its boundary along the Hopf fibration $\partial B^{2 n}(r) \rightarrow \mathbb{C P}^{n-1}$. The resulting space is naturally a smooth symplectic manifold. This can be shown through symplectic cutting $[\mathrm{L}]$ or by an explicit formula on the complex blow-up [GS3]. For more details see [MS1, Section 7.1]. The submanifold $\mathbb{C P}^{n-1}$ is called the exceptional divisor.

Blow-ups of a symplectic manifold. A blow-up of a $2 n$-dimensional symplectic manifold $(M, \omega)$ is a new symplectic manifold that is constructed in the following way. Let $\Omega \subset \mathbb{C}^{n}$ be an open ball centered at the origin of radius greater than $r$, and let $i: \Omega \rightarrow M$ be a symplectomorphism onto an open subset of $M$. The standard blow-up in $\Omega$ of size $r^{2} / 2$ transports to $M$ through $i$.

Let $(\tilde{M}, \tilde{\omega})$ denote the resulting manifold. Then

$$
\operatorname{dim} H_{2}(\tilde{M})=\operatorname{dim} H_{2}(M)+1,
$$

and

$$
\frac{1}{(2 \pi)^{n}} \int_{\tilde{M}} \widetilde{\omega}^{n}=\frac{1}{(2 \pi)^{n}} \int_{M} \omega^{n}-\frac{\epsilon^{n}}{n !}
$$

where $\epsilon=r^{2} / 2$ is the size of the blow-up.

Similarly, $k$ simultaneous blow-ups are obtained from embeddings $i_{1}: \Omega_{1} \rightarrow M$, $\ldots, i_{k}: \Omega_{k} \rightarrow M$ whose images are disjoint.

Notions of size. The size, or normalized symplectic area, of a two dimensional symplectic manifold $(C, \omega)$ is $\frac{1}{2 \pi} \int_{C} \omega$. Our normalization convention for the Fubini-Study form $\omega_{\mathrm{FS}}$ is that the size of $\mathbb{C P}^{1} \subset \mathbb{C P}^{n}, \frac{1}{2 \pi} \int_{\mathbb{C P}^{1}} \omega_{\mathrm{FS}}$, is equal to one. We also define the size of a ball of radius $r$ in $\mathbb{C}^{n}$ to be $r^{2} / 2$. These notions are compatible with the notion of the size of a symplectic blow-up; in a symplectic blow-up of size $\epsilon$, the resulting exceptional divisor is symplectomorphic to $\left(\mathbb{C P}^{n-1}, \epsilon \omega_{\mathrm{FS}}\right)$.

Constraints on blow-ups. The volume of a symplectic manifold gives constraints on the sizes of its symplectic blow-ups: the volume of an embedded ball cannot exceed the volume of the manifold. Sharper constraints are proved using holomorphic techniques. For instance, suppose that we can perform two blow-ups of $\mathbb{C P}^{2}$ of sizes $\epsilon_{1}$ and $\epsilon_{2}$. By the volume constraint, $\epsilon_{1}^{2}+\epsilon_{2}^{2}<1$. By holomorphic constraints, $\epsilon_{1}+\epsilon_{2}<1$; see $[\mathrm{Gr}]$. 
Gromov and McDuff-Polterovich give sharp constraints for the existence of $k \leq 8$ simultaneous blow-ups of $\mathbb{C P}^{2}$ of equal sizes $\epsilon$. There exist such blow-ups if and only if $\epsilon$ satisfies the following conditions. If $k=2,3,4: \epsilon<\frac{1}{2}$. If $k=5,6: \epsilon<\frac{2}{5}$. If $k=7: \epsilon<\frac{3}{8}$. If $k=8: \epsilon<\frac{6}{17}$. See [MP]. According to Biran, for $k \geq 9$, there exist $k$ symplectic blow-ups of equal sizes $\epsilon$ if and only if $\epsilon$ satisfies the volume constraint, $\epsilon<\frac{1}{\sqrt{k}} ;$ see $[\mathrm{Bi}]$.

It is always possible to perform blow-ups of sufficiently small sizes, because, by the Darboux theorem, there always exist Darboux charts, and these contain balls.

Uniqueness of blow-ups of $\mathbf{C P}^{2}$. A manifold obtained from $\mathbb{C P}^{2}$ by a sequence of symplectic blow-ups is determined up to symplectomorphism by the sizes of the blow-ups; see $[\mathrm{McD}]$. We will not use this in proving our theorem.

\section{Equivariant symplectic blow-ups}

Equivariant symplectic blow-ups. The standard action of the unitary group $U(n)$ descends to the standard blow-ups of $\mathbb{C}^{n}$. Let $M$ be a $2 n$-dimensional symplectic manifold with an action of a compact group $G$. Let $\Omega \subset \mathbb{C}^{n}$ be an open ball centered at the origin of radius greater than $r$. Let $i: \Omega \rightarrow M$ be a $G$-equivariant symplectomorphism onto an open subset of $M$, where $G$ acts on $\Omega$ through some homomorphism $G \rightarrow U(n)$. Then the $G$-action naturally extends to the symplectic blow-up of $M$ obtained from $i$. If the action on $M$ is Hamiltonian, its moment map naturally extends to the blow-up. When $G \cong\left(S^{1}\right)^{n}$ we call this a toric blow-up.

If $G$ is Abelian then, after possibly composing the inclusion map $i: \Omega \rightarrow M$ on the right by an element of $U(n)$, we may assume that $G$ acts on $\mathbb{C}^{n}$ by rotations of the coordinates. Specifically, if the action is toric, we may assume that $G$ acts on $\mathbb{C}^{n}$ through an isomorphism with $\left(S^{1}\right)^{n}$, and if $G=S^{1}$, we may assume that its action on $\mathbb{C}^{n}$ is

$$
\lambda \cdot\left(z_{1}, \ldots, z_{n}\right)=\left(\lambda^{m_{1}} z_{1}, \ldots, \lambda^{m_{n}} z_{n}\right)
$$

where $m_{1}, \ldots, m_{n}$ are integers. These integers are called the isotropy weights at $p$; they are determined, up to permutation, by the $S^{1}$-action and the symplectic form.

Size and rational length. The size, or rational length, of an interval $A B$ of rational slope in $\mathbb{R}^{n}$ is the largest positive number $\lambda$ such that the vector $\frac{1}{\lambda} \overrightarrow{A B}$ is in the lattice $\mathbb{Z}^{n}$. This is compatible with the previous notions of "size" (normalized symplectic area); in a symplectic toric manifold $\left(M_{\Delta}, \omega_{\Delta}, \Phi_{\Delta}\right)$ with moment map image $\Delta$, the preimage of an edge of $\Delta$ is a 2-sphere in $M_{\Delta}$ whose normalized symplectic area is equal to the rational length of the edge.

Toric blow-ups in dimension 4. The moment map image of the standard symplectic blow-up of $\mathbb{C}^{2}$ of size $\epsilon$ is shown in Figure 1. It is obtained from the moment map image $\mathbb{R}_{+}^{2}$ of $\mathbb{C}^{2}$ by "chopping off" a triangle whose sides have size $\epsilon$.

A toric blow-up of size $\epsilon$ of a symplectic toric manifold amounts to "chopping off a corner of size $\epsilon$ " of its moment map image $\Delta$. This can be done if and only if there exist two adjacent edges in $\Delta$ whose sizes are both strictly greater than $\epsilon$.

Example. Figure 2 shows the moment map images of toric blow-ups of $\mathbb{C P}^{2}$ of sizes $\epsilon_{1}, \epsilon_{2}, \epsilon_{3}$. By the "uniqueness of blow-ups" of $\mathbb{C P}^{2}$, the resulting manifolds are (nonequivariantly) symplectomorphic. 


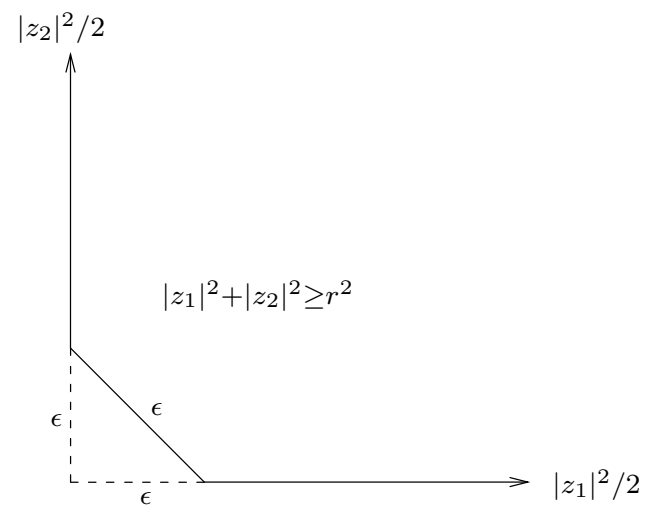

Figure 1. Blow-up of $\mathbb{C}^{2}$ of size $\epsilon=\frac{r^{2}}{2}$
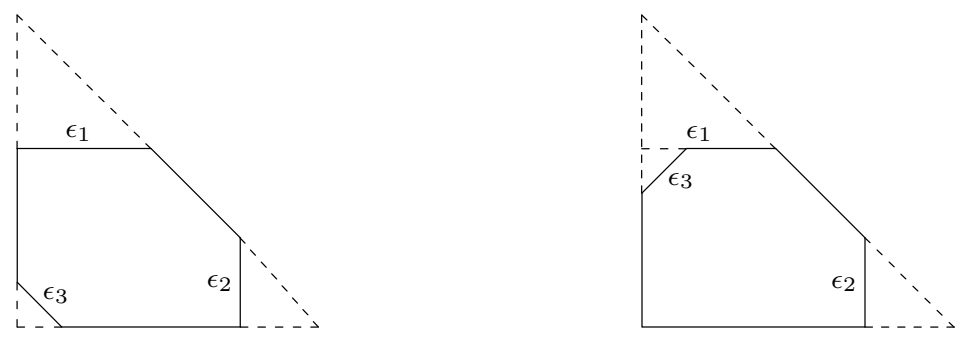

FiguRE 2. Toric blow-ups of sizes $\epsilon_{1}, \epsilon_{2}, \epsilon_{3}$ of $\mathbb{C P}^{2}$

\section{Equal toric blow-ups of $\mathbf{C P}^{2}$.}

Lemma 3.1. $\left(\mathbb{C P}^{2}, \omega_{F S}\right)$ admits a toric blow-up of size $\epsilon>0$ if and only if $\epsilon<1$. $\left(\mathbb{C P}^{2}, \omega_{F S}\right)$ admits two or three toric blow-ups of size $\epsilon>0$ if and only if $\epsilon<\frac{1}{2}$. $\left(\mathbb{C P}^{2}, \omega_{F S}\right)$ does not admit four or more toric blow-ups of equal sizes.

Proof. By Lemma 1.1, the moment map image of $\mathbb{C P}^{2}$ is a triangle in which all edges have size 1. So a toric blow-up of size $\epsilon>0$ can be performed if and only if $\epsilon<1$.

After one such blow-up, the moment map image has edges of sizes $1-\epsilon, \epsilon, 1-\epsilon, 1$ (ordered cyclically), as shown in Figure 3. If $\epsilon \geq \frac{1}{2}$, no two adjacent edges have size greater than $\epsilon$, so one cannot perform a second toric blow-up of size $\epsilon$. If $\epsilon<\frac{1}{2}$, one can perform toric blow-ups of size $\epsilon$ at one or both of the two endpoints of the edge of size 1. The resulting moment map images are shown in Figure 4.

After three toric blow-ups of size $\epsilon$, one out of every two adjacent edges of the moment map image has size $\epsilon$; see Figure 4. So it is impossible to perform another toric blow-up of size $\epsilon$.

Remark 3.2. Let $(M, \omega)$ be a symplectic manifold that is obtained from $\left(\mathbb{C P}^{2}, \omega_{\mathrm{FS}}\right)$ by $k$ simultaneous blow-ups of sizes $\epsilon_{1}, \ldots, \epsilon_{k}$. If $k=1$, then the size of the blow-up must be smaller than 1. If $k \geq 2$, then, by Gromov, $\epsilon_{i}+\epsilon_{j}<1$ for all $i \neq j$. Now suppose that $k \leq 3$. By an argument similar to the proof of Lemma $3.1,\left(\mathbb{C P}^{2}, \omega_{\mathrm{FS}}\right)$ admits toric 


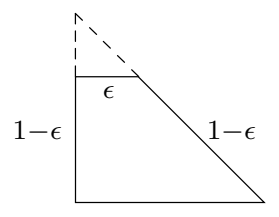

1

Figure 3. One toric blow-up of size $\epsilon$ of $\mathbb{C P}^{2}$
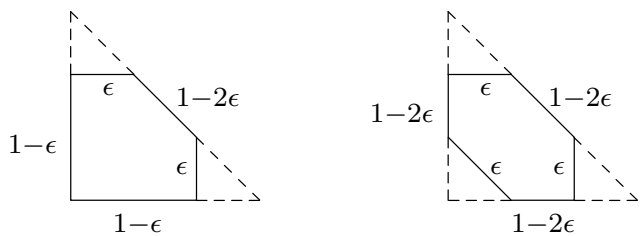

Figure 4. Two or three toric blow-ups of size $\epsilon$ of $\mathbb{C P}^{2}$

blow-ups of the sizes $\epsilon_{1}, \ldots, \epsilon_{k}$. By "uniqueness of blow-ups", the resulting manifold is symplectomorphic to $(M, \omega)$. Thus, any symplectic manifold that is obtained from $\left(\mathbb{C P}^{2}, \omega_{\mathrm{FS}}\right)$ by $k \leq 3$ blow-ups admits a toric (and hence circle) action.

$\mathbf{S}^{1}$-equivariant blow-ups in dimension 4 . In section 1 we defined the labeled graph corresponding to a Hamiltonian $S^{1}$-manifold $(M, \omega, \Phi)$. We will now describe how an equivariant symplectic blow-up affects the graph. To reduce the number of cases that we will need to consider, we will work with extended graphs, obtained from the graph of a Hamiltonian $S^{1}$-manifold by adding edges that are labeled by the integer 1 and that correspond to gradient spheres on which the action is free. (A gradient sphere is a sphere that is invariant under the circle action and under the gradient flow of the moment map with respect to a compatible Riemannian metric. For a generic compatible metric, a gradient sphere can connect an interior fixed point to a minimum or maximum of the moment map, but it cannot connect two interior fixed points; see [Ka1, section 3.2].)

The affect of an equivariant symplectic blow-up is given by Figure 5 in the case of an interior fixed point and by Figures 6- 8 in the case of a fixed point on which the moment map is minimal; the case where the moment map is maximal is similar. These figures are taken from [Ka1]. Such a blow-up can be performed on the manifold if $\lambda>0$ is sufficiently small. The vertices of the resulting graph aquire a natural partial ordering: for any two vertices $v, w$ with moment map labels $\Phi(v), \Phi(w)$, we declare that $v<w$ if and only if $\Phi(v)<\Phi(w)$, and, additionally, either $\Phi(v)$ or $\Phi(w)$ is extremal, or $v$ and $w$ are connected by a chain of edges along which $\Phi$ is monotone.

The operation on graphs that is given by Figures 5-8 can be performed for any positive $\lambda$; we call it a $\lambda$-blow-up of the graph. If $\lambda$ is too large, the moment map labels might be inconsistent with the partial ordering on the vertices, or the area label of a "fat" vertex might become non-positive. We recall Definition 7.1 of [Ka1]: the graph is monotone if 


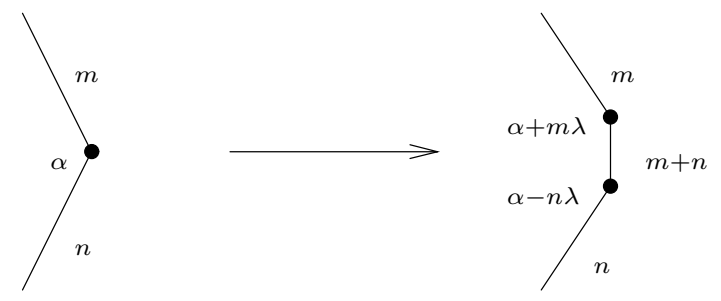

FiguRe 5. $\lambda$-blow-up at interior fixed point
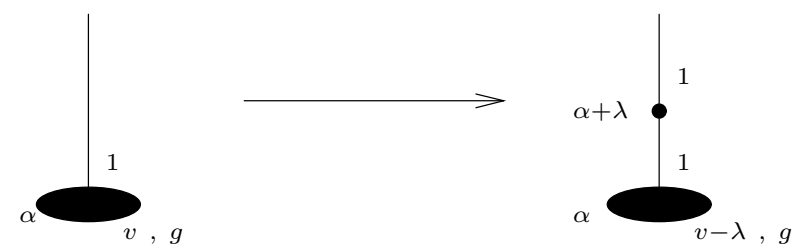

FiguRE 6. $\lambda$-blow-up at non-isolated fixed point on minimum

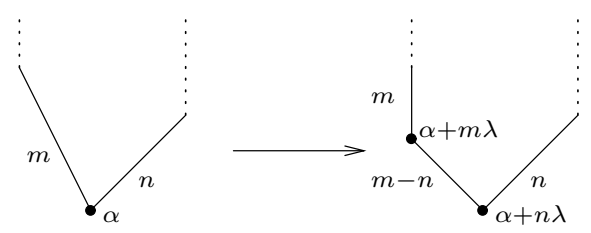

Figure 7. $\lambda$-blow-up at isolated fixed point on minimum with different isotropy weights

(1) its moment map labels are strictly monotone with respect to the partial ordering on its vertices, and

(2) its area labels are positive.

Lemma 3.3. A Hamiltonian $S^{1}$-manifold $(M, \omega, \Phi)$ admits an $S^{1}$-equivariant blowup of size $\epsilon>0$ centred at a fixed point $p \in M^{S^{1}}$ if and only if the following conditions are satisfied.

(1) If $p$ belongs to a $\mathbf{Z}_{k}$-sphere $C$, then $\epsilon<\frac{1}{2 \pi} \int_{C} \omega$.

(2) If $p$ belongs to a two dimensional component $F$ of the fixed point set $M^{S^{1}}$, then $\epsilon<\frac{1}{2 \pi} \int_{F} \omega$.

(3) If $p$ is not a maximum for the moment map, then $\epsilon<\max _{m \in M} \Phi(m)-\Phi(p)$. If $p$ is not a minimum for the moment map, then $\epsilon<\Phi(p)-\min _{m \in M} \Phi(m)$. 


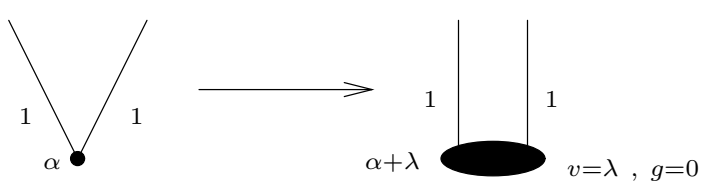

Figure 8. $\lambda$-blow-up at isolated fixed point on minimum with isotropy weights 1,1

(4) If $p$ is an isolated fixed point and is a minimum or maximum for the moment map, and if $q$ is any fixed point other than $p$, then $\epsilon<|\Phi(p)-\Phi(q)|$.

Proof. The conditions in the lemma are equivalent to the $\epsilon$-blown-up graph being monotone; see the discussion between Examples 7.3 and 7.4 of [Ka1]. Proposition 7.2 of [Ka1] asserts

A four dimensional compact Hamiltonian $S^{1}$-space admits an equivariant symplectic blow-up of size $\epsilon$ at some fixed point if and only if the corresponding blown-up graph is monotone.

We sketch why monotonicity is sufficient. By [Ka1, Theorem 7.1], we may assume that the space is Kähler. The $\epsilon$-blown-up graph determines a cohomology class $\Omega$ on the equivariant complex blow-up. Every complex curve is homologous to a positive linear combination of invariant complex curves [Ka1, Lemma C.6]; monotonicity implies that the cohomology class $\Omega$ is positive on invariant complex curves. This, in turn, implies that $\Omega^{2}$ is positive on the fundamental class $[M]$, by [Ka1, Lemma C.4]. Nakai's criterion implies the existence of a Kähler form in the class $\Omega$. This gives a symplectic blow-up of size $\epsilon$, because after blowing-down along the exceptional divisor the graph becomes the same as the initial one. For details, see [Ka1, appendix $\mathrm{C}$ and section 7].

We now show that the conditions are necessary. This argument was omitted from $[\mathrm{Ka} 1]$.

Let $\Omega \subset \mathbb{C}^{2}$ be an open ball of radius $>r$ centered at the origin, where $\epsilon=r^{2} / 2$, and let

$$
i: \Omega \rightarrow M
$$

be a symplectic embedding that is equivariant with respect to a homomorphism $S^{1} \rightarrow$ $U(2)$ and such that $i(0)=p$. Let $m$ and $n$ be the isotropy weights at $p$, so we may assume that the $S^{1}$-action on $\mathbb{C}^{2}$ is

$$
\lambda \cdot\left(z_{1}, z_{2}\right)=\left(\lambda^{m} z_{1}, \lambda^{n} z_{2}\right),
$$

and the moment map on $\mathbb{C}^{2}$ is

$$
\Phi_{\mathbb{C}^{2}}(z)=\Phi(p)+m \frac{\left|z_{1}\right|^{2}}{2}+n \frac{\left|z_{2}\right|^{2}}{2} .
$$

The point $\left(z_{1}, 0\right)$, for $z_{1} \neq 0$, is fixed if and only if $m=0$, and it has stabilizer $\mathbf{Z}_{k}$ if and only if $k=|m| \geq 2$. A similar statement holds for the point $\left(0, z_{2}\right)$. It follows that if $p$ belongs to a $\mathbf{Z}_{k}$-sphere $C$ or to a two dimensional component $F$ of the fixed point set $M^{S^{1}}$ then $i^{-1}(C)$ or $i^{-1}(F)$ is equal to the intersection of $\Omega$ with one of the 
coordinate planes. Because this intersection contains a disk of area $\pi r^{2}=2 \pi \epsilon$, the size of $C$, or $F$, must be greater than $\epsilon$. This immediately implies Conditions (1) and (2).

Condition (3) follows from the fact that the moment map for the $S^{1}$-action on $\mathbb{C}^{2}$ satisfies $\sup _{z \in \Omega} \Phi_{\mathbb{C}^{2}}(z)>\Phi(p)+\epsilon$ if $p$ is not a maximum for the moment map and $\inf _{z \in \Omega} \Phi_{\mathbb{C}^{2}}(z)<\Phi(p)-\epsilon$ if $p$ is not a minimum for the moment map.

Let $p$ be a minimum for the moment map. Then the isotropy weights $m$ and $n$ are non-negative. Let $I$ be an open interval containing the segment $[\Phi(p), \Phi(p)+$ $\min (m \epsilon, n \epsilon)]$ such that $\Phi_{\mathbb{C}^{2}}^{-1}(I)$ is contained in $\Omega$. Consider the commuting diagram

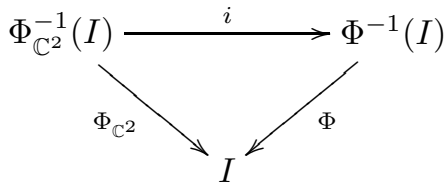

Because the maps to $I$ are proper, the horizontal map $i$ is proper, so its image is closed in $\Phi^{-1}(I)$. Because the map $i$ is a diffeomorphism with an open set, its image is also open. Being the moment map pre-image of a convex set, $\Phi^{-1}(I)$ is connected. Thus, $i: \Phi_{\mathbb{C}^{2}}^{-1}(I) \rightarrow \Phi^{-1}(I)$ is an (equivariant) diffeomorphism, and $\Phi^{-1}(I)$ does not contain fixed points other than $p$. This implies Condition (4) for a minimum. Condition (4) for a maximum is proved in a similar way.

If $\widetilde{M}^{\prime}$ is obtained from $\widetilde{M}$ by an equivariant blow-up, and $q$ is an isolated fixed point in $\widetilde{M}$ different from the point at which the blow-up is centred, then $q$ can also be considered as a point of $\widetilde{M}^{\prime}$. This follows from the fact that the image of an equivariant open embedding $i: \Omega \rightarrow \widetilde{M}$, when $\Omega \subset \mathbb{C}^{n}$ is an open ball centered at the origin, cannot contain an isolated fixed point other than $i(0)$.

Lemma 3.4. Let $(M, \omega, \Phi)$ be a four dimensional compact Hamiltonian $S^{1}$-manifold. Let $(\widetilde{M}, \widetilde{\omega}, \widetilde{\Phi})$ be an equivariant blow-up of $(M, \omega, \Phi)$ of size $\epsilon>0$ centred at an isolated fixed point $p \in M^{S^{1}}$. Let $E \subset \widetilde{M}$ be the resulting exceptional divisor. Then $\widetilde{M}$ does not admit an equivariant symplectic blow-up of size $\epsilon$ that is centred at a fixed point in $E$.

Moreover, suppose that $q \in E$ is an isolated fixed point. Let $\widetilde{M}^{\prime}$ be obtained from $\widetilde{M}$ by a sequence of equivariant symplectic blow-ups at points other than $q$. Then $\widetilde{M}^{\prime}$ does not admit an equivariant symplectic blow-up of size $\epsilon$ that is centred at $q$.

Proof. Let $m$ and $n$ be the isotropy weights at $p$. Because $p$ is an isolated fixed point, $m$ and $n$ are non-zero. Because the action is effective, $m$ and $n$ are relatively prime. The exceptional divisor $E$ is symplectomorphic to $\left(\mathbb{C P}^{1}, \epsilon \omega_{\mathrm{FS}}\right)$ with the $S^{1}$-action $\lambda \cdot[z, w]=\left[\lambda^{m} z, \lambda^{n} w\right]$.

If $k:=|m-n| \geq 2$, then $E$ is a $\mathbf{Z}_{k}$-sphere of size $\epsilon$ passing through $q$. Additional blow-ups transform $E$ into a $\mathbf{Z}_{k}$ sphere through $q$ of size $\leq \epsilon$. The lemma then follows from part (1) of Lemma 3.3.

If $m=n=1$ or $m=n=-1$, then $E \subset \widetilde{M}$ is fixed under the $S^{1}$-action. The lemma then follows from part (2) of Lemma 3.3. 

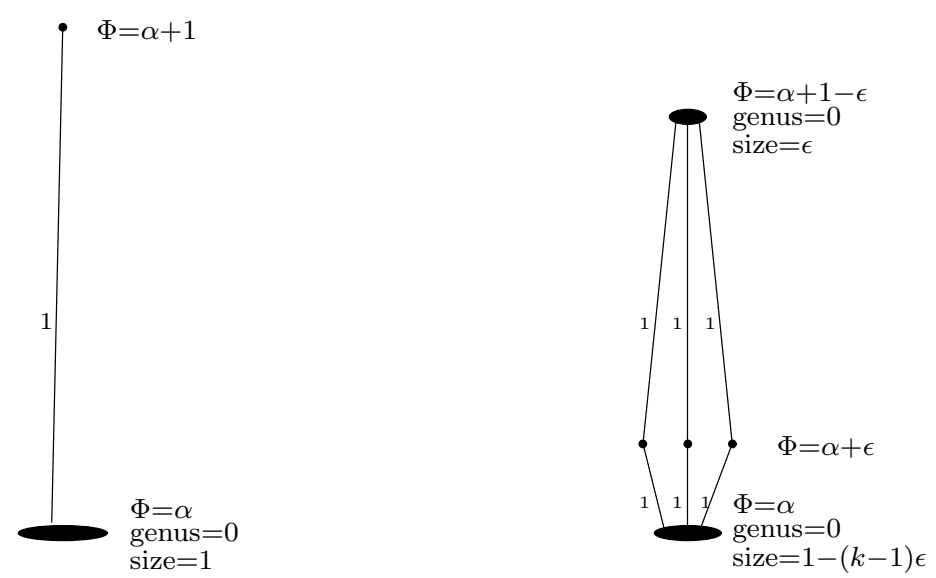

Figure $9 . S^{1}$-equivariant blow-ups of $\mathbb{C P}^{2}$

Now suppose that $|m-n|=1$. Then $m$ and $n$ must have the same sign, and the isolated fixed point $p$ is a minimum or maximum for the moment map $\Phi$. Suppose that it is a minimum; the case of a maximum is similar. Then $E$ connects the minimum of $\widetilde{\Phi}$ with an interior fixed point, and the moment map values at these two points differ by $\epsilon$. First, suppose that $q$ is the interior fixed point on $E$. Because blowing up does not decrease the minimal value of the moment map, part (3) of Lemma 3.3 implies that $\widetilde{M^{\prime}}$ does not admit an equivariant blow-up of size $\epsilon$ centered at $q$. Next, suppose that $q$ is the minimum for $\widetilde{\Phi}$. Since blowing up at an interior fixed point creates two interior fixed points, one of which has a smaller moment map value, part (4) of Lemma 3.3 implies that $\widetilde{M}^{\prime}$ does not admit an equivariant blow-up of size $\epsilon$ centered at $q$.

\section{Equal $\mathbf{S}^{\mathbf{1}}$-equivariant blow-ups of $\mathbf{C P}^{\mathbf{2}}$.}

Lemma 3.5. For $k \geq 2$, $\left(\mathbb{C P}^{2}, \omega_{F S}\right)$ admits an $S^{1}$-action and $k$ equivariant blow-ups of size $\epsilon>0$ if and only if $\epsilon<\frac{1}{k-1}$.

Remark 3.6. Any circle action on $\left(\mathbb{C P}^{2}, \omega_{\mathrm{FS}}\right)$ extends to a toric action; see $[\mathrm{Ka} 2$, Theorem 1]. Thus, by Lemma 3.1 , for any circle action on $\left(\mathbb{C P}^{2}, \omega_{\mathrm{FS}}\right)$, there exists an $S^{1}$-equivariant blow-up of size $\epsilon>0$ if and only if $\epsilon<1$, and, for $k=2$ or $k=3$, for any circle action on $\left(\mathbb{C P}^{2}, \omega_{\mathrm{FS}}\right)$, there exist $k$ equivariant blow-ups of size $\epsilon>0$ if and only if $\epsilon<\frac{1}{2}$.

Proof of Lemma 3.5. The left hand side of Figure 9 shows $\mathbb{C P}^{2}$ with the $S^{1}$-action

$$
a \cdot\left[z_{0}, z_{1}, z_{2}\right]=\left[z_{0}, z_{1}, a z_{2}\right] .
$$

Suppose that $(k-1) \epsilon<1$. Then we can perform one blow-up at the isolated fixed point and $(k-1)$ blow-ups at points on the fixed surface; see Lemma 3.3. The result is shown on the right hand side of Figure 9. 


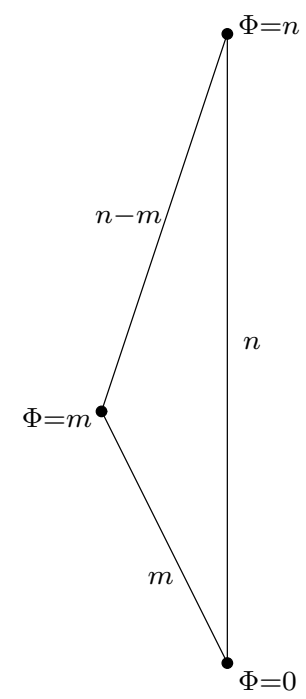

Figure $10 . S^{1}$-action on $\mathbb{C P}^{2}$

From Lemma 3.3 it follows that the condition $(k-1) \epsilon<1$ is necessary for there to exist $k$ equivariant blow-ups of sizes $\epsilon>0$.

A similar argument holds if we start from $\mathbb{C P}^{2}$ with the opposite $S^{1}$-action, $a$. $\left[z_{0}, z_{1}, z_{2}\right]=\left[z_{0}, z_{1}, a^{-1} z_{2}\right]$.

Now suppose that we start with $\mathbb{C P}^{2}$ with any other $S^{1}$-action. By Lemma 1.2, we may assume that the action has the form $\lambda \cdot\left[z_{0}, z_{1}, z_{2}\right]=\left[\lambda^{a} z_{0}, \lambda^{b} z_{1}, \lambda^{c} z_{2}\right]$ for some $a, b, c \in \mathbb{Z}$. After possibly permuting the homogeneous coordinates and multiplying them by the same power of $\lambda$, we may assume that the action is

$$
\lambda \cdot\left[z_{0}, z_{1}, z_{2}\right]=\left[z_{0}, \lambda^{m} z_{1}, \lambda^{n} z_{2}\right]
$$

with $0 \leq m \leq n$. Because the action is effective, $m$ and $n$ are relatively prime. We already covered the case that $m=0$ and $n=1$, and, since $\left[z_{0}, \lambda z_{1}, \lambda z_{2}\right]=$ $\left[\lambda^{-1} z_{0}, z_{1}, z_{2}\right]$, we also covered the case $m=n=1$. So we may assume that $1 \leq m \leq$ $n-1$. This action, with the minimal moment map value $\Phi=0$, is shown in Figure 10 .

By Lemma 3.3, if we perform equivariant blow-ups at two of the three fixed points, the sum of the sizes of the blow-ups is less than 1. Fix $\epsilon>0$. By Lemma 3.4, we can perform equivariant blow-ups of size $\epsilon$ only at the original three fixed points. Thus, if $k$ is the number of blow-ups, then either $k=1$ and $\epsilon<1$, or $k \in\{2,3\}$ and $\epsilon<\frac{1}{2}$. In all these cases, $(k-1) \epsilon<1$.

Figure 11 shows the result of three equivariant blow-ups of size $\epsilon$.

Equivariant symplectic blow-down. Let a compact Lie group $G$ act on a four dimensional symplectic manifold $(M, \omega)$. Let $C \subset M$ be a smooth, $G$-invariant, symplectic 2-sphere, whose homology class satisfies $[C] \cdot[C]=-1$. By the equivariant version of Weinstein's tubular neighborhood theorem [W], a neighborhood of $C$ is equivariantly symplectomorphic to a neighborhood of the exceptional divisor in a 


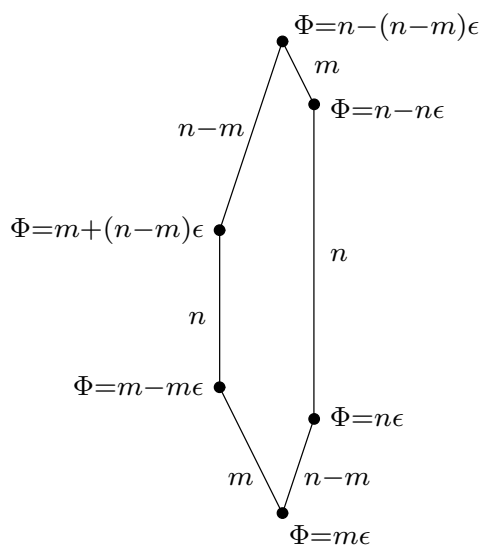

Figure 11. Three $S^{1}$-equivariant blow-ups of $\mathbb{C P}^{2}$

standard blow-up of $\mathbb{C}^{2}$. We can then equivariantly blow-down $M$ along $C$. This yields a symplectic manifold $\left(N, \omega_{N}\right)$ with a $G$-action whose equivariant blow-up is $(M, \omega)$.

\section{Circle and torus actions on equal symplectic blow-ups of $\mathrm{CP}^{2}$.}

We are now ready to state our main result. Recall that we assume that our circle or torus actions are always effective.

Theorem 4.1. Let $\epsilon=\frac{1}{n}$, where $n$ is a natural number. Let $\left(M_{k}, \omega_{\epsilon}\right)$ be a symplectic manifold that is obtained from $\left(\mathbb{C P}^{2}, \omega_{F S}\right)$ by $k \geq 4$ simultaneous blow-ups of size $\epsilon$.

(1) $\left(M_{k}, \omega_{\epsilon}\right)$ does not admit a two dimensional torus action.

(2) If $\epsilon \geq \frac{1}{k-1}$ then $\left(M_{k}, \omega_{\epsilon}\right)$ does not admit a circle action.

Corollary 4.2. For the following values of $k$ and $\epsilon$, it is possible to perform $k$ simultaneous symplectic blow-ups of $\left(\mathbb{C P}^{2}, \omega_{F S}\right)$ of equal sizes $\epsilon$, but the resulting manifold $\left(M_{k}, \omega_{\epsilon}\right)$ does not admit a circle action.

\begin{tabular}{c|c}
$k$ & $\epsilon$ \\
\hline 4 & $\frac{1}{3}$ \\
\hline 5 & $\frac{1}{3}, \frac{1}{4}$ \\
\hline 6 & $\frac{1}{3}, \frac{1}{4}, \frac{1}{5}$ \\
\hline 7 & $\frac{1}{n}$, where $3 \leq n \leq 6$ \\
\hline 8 & $\frac{1}{n}$, where $3 \leq n \leq 7$ \\
\hline$\geq 9$ & $\frac{1}{n}$, where $\sqrt{k}<n \leq k-1$ \\
\hline
\end{tabular}

This follows from the sharpness of the constraints on blow-ups listed in section 2 and from Theorem 4.1.

Remark 4.3. The reason that Theorem 4.1 does not follow directly from Lemmas 3.1 and 3.5 is that a-priori $\left(M_{k}, \omega_{\epsilon}\right)$ might admit an action which does not arise from an action on $\mathbb{C P}^{2}$ by performing the blow-ups equivariantly. To show that such 
exotic actions do not exist, we apply Gromov's theory of holomorphic curves. This application is easiest in the case $\epsilon=\frac{1}{n}$.

Remark 4.4. The reason that we require the blow-ups to be simultaneous is that in this case the exceptional divisors are disjoint symplectically embedded spheres; see Lemma 6.1. In fact, by "uniqueness of blow-ups", a manifold obtained by a sequence of blow-ups is symplectomorphic to one that is obtained by simultaneous blow-ups.

\section{Holomorphic spheres}

In this section we recall some well-known results from Gromov's theory of holomorphic curves. We restrict our attention to curves of genus zero. Wherever we do not specify the degree of smoothness, we assume that maps are $C^{\infty}$ smooth and spaces of maps are equipped with the $C^{\infty}$ topology.

An almost complex structure on a manifold $M$ is an automorphism $J: T M \rightarrow T M$ of the tangent bundle such that $J^{2}=-$ identity. It is compatible with a symplectic form $\omega$ if $\langle u, v\rangle:=\omega(u, J v)$ is symmetric and positive definite. This implies that for every embedded submanifold $C \subset M$, if $J(T C)=T C$ then $\left.\omega\right|_{T C}$ is non-degenerate. On a symplectic manifold there always exists a compatible almost complex structure. If a symplectic manifold admits an action of a compact Lie group $G$, there always exists a compatible almost complex structure that is $G$-invariant.

Fix a compact symplectic manifold $(M, \omega)$. Let $\mathcal{J}$ denote the space of almost complex structures $J$ on $M$ that are compatible with $\omega$. The space $\mathcal{J}$ is contractible [MS1, Prop. 2.50(iii)]; in particular, it is connected. The first Chern class of the complex vector bundle $(T M, J)$ is independent of the choice of $J \in \mathcal{J}$; we denote it $c_{1}(T M)$.

Fix $J \in \mathcal{J}$. A parametrized $J$-sphere is a map $f: \mathbb{C P}^{1} \rightarrow M$ which is $J$-holomorphic, that is, which satisfies the Cauchy-Riemann equations $d f \circ j=J \circ d f$. It is called simple if it cannot be factored through a branched covering of $\mathbb{C P}^{1}$. An embedding is a one-to-one immersion which is a homeomorphism with its image. An embedded $J$-sphere $C \subset M$ is the image of a $J$-holomorphic embedding $f: \mathbb{C P}^{1} \rightarrow M$. In particular, such a $C$ is an embedded symplectic sphere.

Let $A \in H_{2}(M ; \mathbb{Z})$ be a homology class. For $J \in \mathcal{J}$, let $\mathcal{M}(A, J)$ denote the set of simple parametrized $J$-spheres in the class $A$. Notice that if $A \cdot A=-1$ then every parametrized $J$-sphere in the class $A$ is simple. Consider the universal moduli space

$$
\mathcal{M}(A, \mathcal{J})=\{(f, J) \mid J \in \mathcal{J}, f \in \mathcal{M}(A, J)\} .
$$

(In the notation of McDuff and Salamon, [MS3, §3.1], this space would be denoted by $\mathcal{M}^{*}(A, \Sigma ; \mathcal{J})$ with $\Sigma=\mathbb{C P}^{1}$.)

Lemma 5.1. Let $(M, \omega)$ be a compact symplectic four-manifold. Let $A \in H_{2}(M ; \mathbb{Z})$ be a homology class. Let $J \in \mathcal{J}$ be an almost complex structure. Suppose that $c_{1}(T M)(A) \geq 1$ and that there exists an immersed $J$-sphere $f_{0}: \mathbb{C P}^{1} \rightarrow M$ in the class $A$. Then the image of the projection map $\pi: \mathcal{M}(A, \mathcal{J}) \rightarrow \mathcal{J}$ contains a neighborhood $\Omega$ of $J$ in $\mathcal{J}$.

Proof. Let $\ell \geq 2$. Let $\mathcal{J}^{\ell}$ denote the space of almost complex structures on $M$ of type $C^{\ell}$ that are compatible with $\omega$, and let $\mathcal{M}\left(A, \mathcal{J}^{\ell}\right)$ denote the space of pairs $(f, J)$ where $J \in \mathcal{J}^{\ell}$ and where $f: \mathbb{C P}^{1} \rightarrow M$ is a $J$-holomorphic map of type $C^{\ell}$ in the class $A$ that cannot be factored through a branched covering of $\mathbb{C P}^{1}$. Let 
$\pi^{\ell}: \mathcal{M}\left(A, \mathcal{J}^{\ell}\right) \rightarrow \mathcal{J}^{\ell}$ denote the projection map. Then $\mathcal{J}^{\ell}$ and $\mathcal{M}\left(A, J^{\ell}\right)$ are Banach manifolds and $\pi^{\ell}$ is differentiable; see [MS3, Proposition 3.2.1]. The assumptions that $c_{1}(T M)(A) \geq 1$ and that $f_{0}$ is an immersion imply that $\left.d \pi^{\ell}\right|_{\left(f_{0}, J\right)}$ is onto; see the regularity criterion in Lemma 3.3.3 of [MS3], and see Remark 3.2.8 of [MS3]. Also, the kernel of $\left.d \pi^{\ell}\right|_{\left(f_{0}, J\right)}$ is finite dimensional; see the proof of Theorem 3.1.5 on p. 51-52 of [MS3]. In particular, the kernel splits, that is, it has a closed complementary subspace in $T_{(f, J)} \mathcal{M}\left(A, \mathcal{J}^{\ell}\right)$; see [La, p. 4]. By the implicit function theorem for Banach spaces, there exist a neighborhood of $(f, J)$ in $\mathcal{M}\left(A, \mathcal{J}^{\ell}\right)$ in which $\pi^{\ell}$ is a projection map; in particular, the image of $\pi^{\ell}$ contains an open neighborhood $\Omega^{\ell}$ of $J$ in $\mathcal{J}^{\ell}$; see [La, Chapter I, $\S 5$, Cor. 2s]. Since the $C^{\ell}$ topology on $\mathcal{J} \subset \mathcal{J}^{\ell}$ is coarser then the $C^{\infty}$ topology, and $\Omega^{\ell}$ is open in $\mathcal{J}^{\ell}$, the intersection $\Omega:=\Omega^{\ell} \cap \mathcal{J}$ is open in $\mathcal{J}$. Let $J^{\prime} \in \Omega$. Since $J^{\prime} \in \Omega^{\ell}$, there exists a $J^{\prime}$-holomorphic sphere $f: \mathbb{C P}^{1} \rightarrow M$ of type $C^{\ell}$ in the class $A$. Since $J^{\prime}$ is smooth, by "elliptic regularity", $f$ is smooth; see [MS3, Proposition 3.1.9]. The lemma follows.

The group $\operatorname{PSL}(2, \mathbb{C})$ of Möbius transformations of $\mathbb{C P}^{1}$ naturally acts on $\mathcal{M}(A, \mathcal{J})$ by reparameterizations. We say that $A$ is indecomposable if it cannot be written as a sum $A=A_{1}+A_{2}$ where $A_{i} \in H_{2}(M ; \mathbb{Z})$ and $\int_{A_{i}} \omega>0$.

Recall that a continuous map between topological spaces is called proper if and only if the pre-image of any compact set is compact.

Lemma 5.2. If $A$ is indecomposable then the map

$$
\mathcal{M}(A, \mathcal{J}) / \operatorname{PSL}(2, \mathbb{C}) \longrightarrow \mathcal{J}
$$

that is induced from $\pi$ is proper.

Proof. By Gromov's compactness theorem [MS3, Theorem 5.3.1], since $A$ is indecomposable, if $J_{n}$ converges in $\mathcal{J}$, then every sequence $\left(f_{n}, J_{n}\right)$ in $\mathcal{M}(A, \mathcal{J})$ has a convergent sub-sequence. It follows that the map (5.1) is proper.

Lemma 5.3. Suppose that $\operatorname{dim} M=4$. Let $A \in H_{2}(M ; \mathbb{Z})$ be a homology class which is represented by an embedded symplectic sphere $C$. Then

(1) There exists an almost complex structure $J_{0} \in \mathcal{J}$ for which $C$ is a $J_{0}$-sphere.

(2) For any $J \in \mathcal{J}$ and any parametrized $J$-sphere $f: \mathbb{C P}^{1} \rightarrow M$ in the class $A$, the map $f$ is an embedding.

Proof. Construct $J_{0}$ as follows. Let $f_{0}: \mathbb{C P}^{1} \rightarrow M$ be a symplectic embedding whose image is $C$. Define $\left.J_{0}\right|_{T C}$ such that $f_{0}$ is holomorphic. Extend it to a compatible fiberwise complex structure on the symplectic vector bundle $\left.T M\right|_{C}$. Then extend it to a compatible almost complex structure on $M$. See [MS1, Section 2.6]. Then $\left(f_{0}, J_{0}\right) \in \mathcal{M}(A, \mathcal{J})$.

By the adjunction inequality, for any $(f, J) \in \mathcal{M}(A, \mathcal{J})$,

$$
A \cdot A-c_{1}(T M)(A)+2 \geq 0,
$$

with equality if and only if $f$ is an embedding; see [MS3, Cor. E.1.7]. Applying this to $\left(f_{0}, J_{0}\right)$, we get that the homology class $A$ satisfies $A \cdot A-c_{1}(T M)(A)+2=0$. Applying the adjunction inequality to any other $(f, J) \in \mathcal{M}(A, \mathcal{J})$, we get that $f$ is an embedding. 
Lemma 5.4. Let $(M, \omega)$ be a compact symplectic four-manifold. Let $A \in H_{2}(M ; \mathbb{Z})$ be an indecomposable homology class which is represented by an embedded symplectic sphere and such that $c_{1}(T M)(A) \geq 1$. Then for any almost complex structure $J \in \mathcal{J}$ there exists an embedded $J$-sphere in the class $A$.

Proof. Let $C \subset M$ be an embedded symplectic sphere such that $[C]=A$. By part 1 of Lemma $5.3, \mathcal{M}(A, \mathcal{J})$ is non-empty. So the image of $\pi$ is a non-empty subset of $\mathcal{J}$.

Let $(f, J) \in \mathcal{M}(A, \mathcal{J})$. By part 2 of Lemma 5.3, $f$ is an embedding. By Lemma 5.1, the image of $\pi$ contains a neighborhood $\Omega$ of $J$ in $\mathcal{J}$. Thus, the map $\pi$ is open. In particular, the image of $\pi$ is an open subset of $\mathcal{J}$.

The image of $\pi$ is equal to the image of the induced map (5.1). Because, by Lemma 5.2 , this map is proper, its image is a closed subset of $\mathcal{J}$.

We have shown that the image of $\pi$ is a subset of $\mathcal{J}$ which is nonempty, open, and closed. Because $\mathcal{J}$ is connected, it follows that $\pi$ is onto. This proves the Lemma.

\section{Proof of the main result}

Lemma 6.1. Let $\epsilon=\frac{1}{n}$ where $n$ is a natural number. Let $\left(M_{k}, \omega_{\epsilon}\right)$ be a symplectic manifold that is obtained from $\left(\mathbb{C P}^{2}, \omega_{F S}\right)$ by $k$ simultaneous blow-ups of size $\epsilon$. Let $E_{i} \in H_{2}\left(M_{k} ; \mathbb{Z}\right)$, for $i=1, \ldots, k$, be the homology classes of the exceptional divisors. Let a compact connected Lie group $G$ act on $\left(M_{k}, \omega_{\epsilon}\right)$ symplectically. Then there exist pairwise disjoint $G$-invariant embedded symplectic spheres $C_{1}, \ldots, C_{k}$ in $M_{k}$ whose homology classes are $\left[C_{i}\right]=E_{i}$.

Proof. Let $C_{i}^{0}$ be the exceptional divisors in $\left(M_{k}, \omega_{\epsilon}\right)$, so that $E_{i}=\left[C_{i}^{0}\right]$. Each $C_{i}^{0}$ is an embedded symplectic sphere.

The second homology group $H_{2}\left(M_{k} ; \mathbb{Z}\right)$ is generated by $\left[\mathbb{C P}^{1}\right]$ and the $E_{i}$ 's. For each $A \in H_{2}\left(M_{k} ; \mathbb{Z}\right)$, the size $\frac{1}{2 \pi} \int_{A} \omega$ is an integer multiple of $\frac{1}{n}$. It follows that any homology class whose size is equal to $\frac{1}{n}$ is indecomposable. In particular, each of the classes $E_{i}$ is indecomposable.

Let $J_{G}$ be a $G$-invariant almost complex structure on $M_{k}$ that is compatible with $\omega_{\epsilon}$. By Lemma 5.4, for each $i$ there exists an embedded $J_{G}$-sphere $C_{i} \subset M_{k}$ such that $\left[C_{i}\right]=E_{i}$. We will now show

(1) $C_{1}, \ldots, C_{k}$ are disjoint;

(2) each $C_{i}$ is $G$-invariant;

(3) each $C_{i}$ is symplectic.

For $i \neq j$, because $E_{i} \neq E_{j}$, the spheres $C_{i}$ and $C_{j}$ do not coincide. By positivity of intersections, and since $E_{i} \cdot E_{j}=0$, the spheres $C_{i}$ and $C_{j}$ are disjoint.

Let $a \in G$. Because $G$ is connected, $\left[a C_{i}\right]=\left[C_{i}\right]=E_{i}$. By positivity of intersections and since $E_{i} \cdot E_{i}=-1, a C_{i}$ and $C_{i}$ must coincide.

Because $C_{i}$ is an embedded $J_{G}$-sphere and $J_{G}$ is compatible by $\omega_{\epsilon}, C_{i}$ is symplectic.

Remark 6.2. The assertion of Lemma 6.1 is false when $\frac{1}{2}<\epsilon<1$ and $k=1$. For a detailed example see [Ke1, Section 6] or [Ke2].

Proof of Theorem 4.1. Let $\epsilon=\frac{1}{n}$ where $n$ is a natural number. Let $\left(M_{k}, \omega_{\epsilon}\right)$ be a symplectic manifold that is obtained from $\left(\mathbb{C P}^{2}, \omega_{\mathrm{FS}}\right)$ by $k$ simultaneous blow-ups of size $\epsilon$. 
Let $G \cong\left(S^{1}\right)^{2}$ or $G \cong S^{1}$ act on $\left(M_{k}, \omega_{\epsilon}\right)$; because $M_{k}$ is simply connected, the action is Hamiltonian.

Let $C_{i}$ be the spheres obtained from Lemma 6.1. Perform equivariant symplectic blow-downs of $\left(M_{k}, \omega_{\epsilon}\right)$ along the $C_{i}$ 's. By (2.1) and (2.2), each such a blow-down decreases the second Betti number by one and increases the symplectic volume by $(2 \pi)^{2} \epsilon^{2} / 2$.

We get a four dimensional symplectic manifold $\left(N, \omega_{N}\right)$, with an effective Hamiltonian $G$-action, $\operatorname{dim} H_{2}(N)=1$ and the volume of $N$ is the same as that of $\left(\mathbb{C P}^{2}, \omega_{\mathrm{FS}}\right)$. By Lemmas 1.1 and 1.2 , this manifold is equivariantly symplectomorphic to $\mathbb{C P}^{2}$ with its standard symplectic form. Viewing this process in reverse order, we get that $\left(M_{k}, \omega_{\epsilon}\right)$ is obtained from $\left(\mathbb{C P}^{2}, \omega_{\mathrm{FS}}\right)$ by $k$ equivariant blow-ups of size $\epsilon$.

The theorem now follows from Lemmas 3.1 and 3.5.

Remark 6.3. Scott Baldridge [B1] has shown that any four-manifold that admits a symplectic structure and a circle action with fixed points must be rational or ruled. Consequently, such a manifold admits a symplectic form that is preserved by a circle action. Baldridge asked [B2] whether any symplectic form on such a manifold is preserved by some circle action. Our result shows that the answer is "no". For example, the manifold obtained from $\mathbb{C P}^{2}$ by four blow-ups admits circle actions and admits symplectic forms; by $[\mathrm{T}]$, this manifold admits a symplectic form such that each of the four exceptional divisors has size $1 / 3$; by Theorem 4.1 , this symplectic form is not preserved under any circle action.

\section{Acknowledgement}

Most of the work on this project has taken place when the authors were affiliated with the Hebrew University of Jerusalem.

We are grateful to Paul Biran, Francois Lalonde, Dusa McDuff, Martin Pinsonnault, Leonid Polterovich, and Dietmar Salamon for valuable discussions. We are grateful to the referee for thoughtful comments.

\section{References}

[At] M. F. Atiyah, Convexity and commuting Hamiltonians, Bull. London Math. Soc. 14 (1982), no. $1,1-15$

[Au] M. Audin, The topology of torus actions on symplectic manifolds, Progress in Math. 93, Birkhäuser Verlag, Basel, 1991; Second Edition: 2004.

[B1] S. Baldridge, Seiberg-Witten vanishing theorem for $S^{1}$-manifolds with fixed points, Pacific Journal of Math. 217 (2004), no. 1, 1-10.

[B2] S. Baldridge, private communication.

[Bi] P. Biran, Symplectic packing in dimension 4, Geom. Funct. Anal. 7 (1997), no. 3, 420-437.

[De] T. Delzant, Hamiltoniens périodiques et image convexe de l'application moment, Bulletin de la Société Mathématique de France 116 (1988), no. 3, 315-339.

[GGK] V. Ginzburg, V. Guillemin, and Y. Karshon, Moment maps, Cobordisms, and Hamiltonian Group Actions, Amer. Math. Soc. Math. Surveys and Monographs 98, 2002.

[Gr] M. Gromov, Pseudo holomorphic curves in symplectic manifolds, Inv. Math. 82 (1985), no. $2,307-347$.

[G] V. Guillemin, Moment maps and combinatorial invariants of Hamiltonian $T^{n}$-spaces, Birkhäuser Boston, Boston, MA, 1994.

[GLS] V. Guillemin, E. Lerman, and S. Sternberg, Symplectic Fibrations and Multiplicity Diagrams, Cambridge University Press, 1996. 
[GS1] V. Guillemin and S. Sternberg, Convexity properties of the moment mapping, Invent. Math. 67 (1982), no. 3, 491-513.

[GS2] V. Guillemin and S. Sternberg, Geometric quantization and multiplicities of group representations, Invent. Math. 67 (1982), no. 3, 515-538.

[GS3] V. Guillemin and S. Sternberg, Birational equivalence in the symplectic category, Invent. Math. 97 (1989), no. 3, 485-522.

[HLS] H. Hofer, V. Lizan, and J-C. Sikorav, On genericity for holomorphic curves in fourdimensional almost-complex manifolds, J. Geom. Anal. 7 (1997), no. 1, 149-159.

[Ka1] Y. Karshon, Periodic Hamiltonian flows on four dimensional manifolds, Memoirs of the Amer. Math. Soc. vol. 141 (1999), no. 672.

[Ka2] Y. Karshon, Maximal tori in the symplectomorphism groups of Hirzebruch surfaces, Math. Research Letters 10 (2003), no. 1, 125-132.

[Kel] J. L. Kelley, General topology, Graduate Texts in Mathematics, Spring-Verlag, 1955.

[Ke1] L. Kessler, Torus actions on small blow ups of $\mathbb{C P}^{2}$, Ph.D. thesis, The Hebrew University of Jerusalem, 2004.

[Ke2] L. Kessler, Torus actions on small blow ups of $\mathbb{C P}^{2}$, in preparation.

[La] S. Lang, Differentiable manifolds, John Wiley \& Sons, inc., 1962.

[L] E. Lerman, Symplectic cuts, Math. Research Letters 2 (1995), no. 3, 247-258.

[LT] E. Lerman and S. Tolman, Hamiltonian torus actions on symplectic orbifolds and toric varieties, Trans. Amer. Math. Soc. 349 (1997), no. 10, 4201-4230.

$[\mathrm{McD}]$ D. McDuff, From symplectic deformation to isotopy, in: Topics in symplectic 4-manifolds (Irvine, CA, 1996), 85-99, First int. Press Lect. Ser., I, internat. Press, Cambridge, MA, 1998.

[MS1] D. McDuff and D. Salamon, Introduction to symplectic topology, Oxford University Press, 1998.

[MS2] D. McDuff and D. Salamon, J-holomorphic curves and quantum cohomology, Amer. Math. Soc. Univ. Lect. Ser. 6, 1994.

[MS3] D. McDuff and D. Salamon, J-holomorphic curves and symplectic topology, Amer. Math. Soc. Collo. Pub. 52, 2004.

[MP] D. McDuff and L. Polterovich, Symplectic packings and algebraic geometry, Invent. Math. 115 (1994), no. 3, 405-434.

[T] L. Traynor, Symplectic packing constructions, Journal of Differential Geometry 42 (1995), no. $2,411-429$.

[W] A. Weinstein, Symplectic manifolds and their Lagrangian submanifolds, Adv. Math. 6 (1971), 329-346.

Department of Mathematics, University of Toronto, Toronto, ON M5S 2E4, Canada.

E-mail address: karshon@math.toronto.edu

Courant Institute of Mathematical Sciences, New York University, New York, Ny 10012, U.S.A.

Current address: Department of Mathematics, Massachusetts Institute of Technology, Cambridge, MA 02139, U.S.A.

E-mail address: kessler@math.mit.edu 